\title{
A Note on Normalised Distributions of DC Partial Microdischarges
}

T. Ficker, J. Macur

Statistical distributions (exponential and Pareto) of DC partial microdischarges running within sandwich electrode systems are discussed from the viewpoint of a normalisation procedure which may influence some features of the final distribution.

Keywords: exponential and statistical distributions, partial microdischarges, normalisation procedure.

\section{Introduction}

When studying the statistics of partial microdischarges within sandwich electrode systems loading by dc voltages in excess of Paschen breakdown values, highly asymmetric distributions can be encountered [1]-[4] in both time and height domains.

Time statistics, i.e. the densities of probability $w^{*}(t)$ of time intervals $t$ between microdischarge pulses, follow an exponential distribution

$$
w_{e}^{*}(t)=a \cdot \mathrm{e}^{-a t}, \quad t \in\langle 0, \infty), \quad a=\text { const. }
$$

while the heights $U$ of microdischarge pulses (their peak values) obey a power law of the Pareto type (Pareto distribution of the first kind) [5]

$$
w_{p}(U)=c \cdot U^{-\alpha}, \quad U \in(0, \infty), \quad \alpha>0 .
$$

Some problems may arise when normalised forms of these highly asymmetric distributions should be used, especially with Pareto's distribution (2), certain probability moments of which diverge

$$
\int_{0}^{\infty} w_{p}(U) \mathrm{d} U=\infty .
$$

The goal of this paper is to discuss problems connected with the normalisation of Pareto's statistic (2).

\section{Normalising exponential distribution}

In fact there is no principal problem with normalisation of a measured (unnormalised) exponential distribution

$$
w_{e}(t)=b \cdot \mathrm{e}^{-a t}, \quad b \neq a, \quad S_{e}=\int_{0}^{\infty} w_{e}(t) \mathrm{d} t=\frac{b}{a} \neq 1 .
$$

Since there is no singular point in the interval $\langle 0, \infty)$, normalisation form (1) can easily be found

$$
w_{e}^{*}(t)=\frac{b}{S_{e}} \mathrm{e}^{-a t} .
$$

To verify the exponential character of a function, the semilogarithmic co-ordinates are usually employed. But what will happen with the shape and position of the graph of an exponential function plotted in the semilogarithmic system when the normalising procedure (5) is performed? This is nicely seen from the following two equations, (6) and (7), which were obtained after logarithmic operations had been applied to Eqs. (1) and (5)

$$
\begin{aligned}
\ln w_{e}(t) & =\ln b-a t \Rightarrow y=k-a t, \\
\ln w_{e}^{*}(t) & =\ln b-\ln S_{e}-a t \Rightarrow y^{*}=k^{*}-a t, \\
k^{*} & =k-\ln S_{e} .
\end{aligned}
$$

Therefore, after normalisation the graph of the exponential distribution function plotted in a semilogarithmic system will conserve its shape (straight line), but it will shift its position in the vertical direction by a constant value $\ln S_{e}$. In fact, there is no need to look for the value of $S_{e}$ to obtain a normalisation form (5), since the normalisation constant $b / S=a$ is an invariant appearing in the argument of unnormalised function (4). Fig. 1 shows an unnormalised distribution in semilogarithmic co-ordinates with the following fitting equation

$$
y=0.0685-0.0895 x
$$

which enables us straightforwardly to determine the corresponding normalised exponential distribution

$$
w_{e}^{*}(t)=0.0895 \cdot \mathrm{e}^{-0.0895 t}(\mathrm{~m} \mathrm{~s})^{-1} .
$$

$\ln \left[\mathrm{w} /(\mathrm{ms})^{-1}\right]$

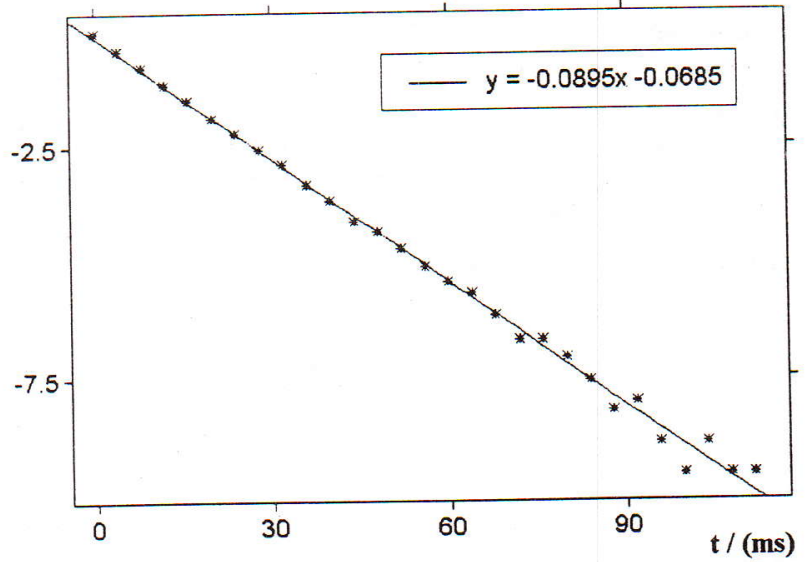

Fig. 1: Probability density of time intervals between microdischarge pulses [4] 


\section{Normalising Pareto distribution}

The situation with the Pareto distribution (2) is more problematic. Due to the singularity at the point $U=0$, the integral (3) over the interval $\langle 0, \infty)$ diverges, and normalisation is not possible, in this interval. Does this mean that normalisation of the Pareto distribution is not possible at all? Normalisation of Pareto's distribution is certainly possible but in an interval $\left(U_{1}, U_{2}\right)$ lacking any singularity, i.e. $U_{1}>0$. Real measurements are usually performed in such intervals. In addition, the intervals in real experiments are usually finite, i.e. $U_{2}<\infty$. For such cases the normalised distribution can be introduced as follows

$$
\begin{gathered}
w_{p}^{*}(U)=\frac{c}{S_{p}} U^{-\alpha}=\frac{(\alpha-1)}{\frac{1}{U_{1}^{\alpha-1}}-\frac{1}{U_{2}^{\alpha-1}} U^{-\alpha},} \\
S_{p}=\int_{U_{1}}^{U_{2}} w_{p}(U) \mathrm{d} U=\frac{c}{1-\alpha}\left(U_{2}^{1-\alpha}-U_{1}^{1-\alpha}\right)
\end{gathered}
$$

For a half-infinite interval $\left(U_{1}, \infty\right)$ it holds

$$
w_{p}(U)=(\alpha-1) U_{1}^{\alpha-1} \cdot U^{-\alpha} .
$$

Analysing the Pareto unnormalised (2) and normalised (11) distributions in bilogarithmic co-ordinates

$$
\begin{aligned}
& \ln w_{p}=\ln c-\alpha \ln U \Rightarrow y=k-\alpha x, \\
& \ln w_{p}^{*}=\ln c-\ln S_{p}-\alpha \ln U \Rightarrow y^{*}=k^{*}-\alpha x, \\
& \quad k^{*}=k-\ln S_{p},
\end{aligned}
$$

similar conclusions as for exponential distribution can be drawn: in bilogarithmic systems the normalisation procedure changes only the vertical position of the graph of the Pareto distribution and does not influence its functional character (slope or asymmetricity). Fig. 2 shows an unnormalised Pareto distribution within the interval $U \in(0.5,5) \mathrm{mV}$ in bilogarithmic co-ordinates with the following fitting equation

$$
y=-0.514-1.42 x
$$

which again enables us to determine the corresponding normalised form of the Pareto distribution. According to (11) we can find

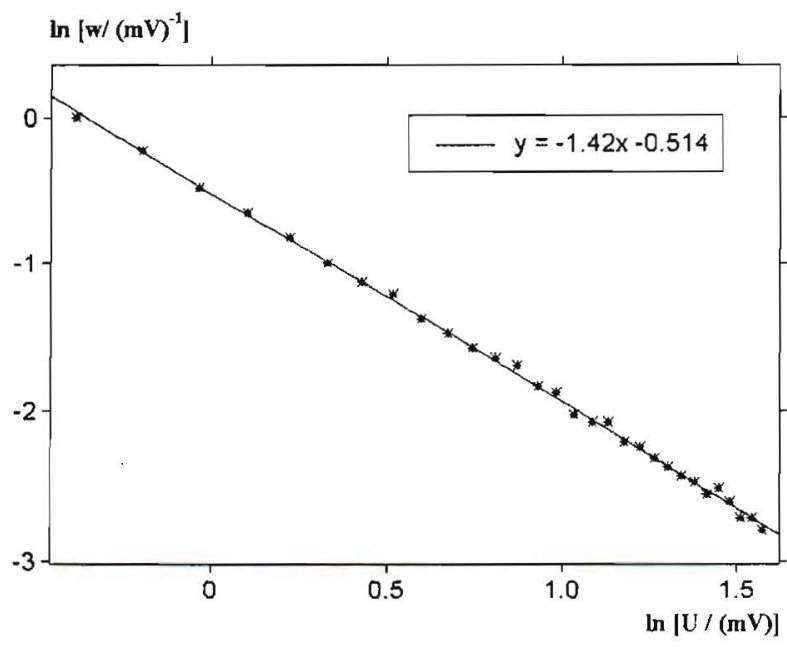

$$
w_{p}^{*}(U)=0.506 \cdot U^{-1.42}(\mathrm{mV})^{-1}
$$

\section{Conclusion}

The Pareto distribution can be normalised in a standard, when the used interval $\left(U_{1}, U_{2}\right)$ does not possesses zero point, i.e. $U_{1}>0$. When plotting both the distributions, i.e. exponential and Pareto, in logarithmic co-ordinates (semi- and bi- systems, respectively), normalisation procedure causes a certain shift of their graphs in the vertical direction, while their shapes and asymmetricities remain unchanged. Their functional character is not influenced by a normalisation procedure. In other words, the normalisation procedure cannot change the results of physical processes and this fact also supports the employment of unnormalised distributions in statistical studies realised in physics and other fields of science and technology.

\section{Acknowledgement}

This work has been supported by the Grant Agency of the Czech Republic under the grant No. 202/03/0011.

\section{References}

[1] Ficker, T.: Fractal Statistics of Partial Discharges with Polymeric Samples. J. Appl. Phys, Vol. 78, 1995, p. 5289-5295.

[2] Fromm, U.: Interpretation of Partial Discharges at DC Voltages. IEEE Trans. Diel. El. Insul., Vol. 2, 1995, p. $761-770$.

[3] Ficker, T., Macur, J., Pazdera, L., Kliment, M., Filip, S.: Simplified Digital Acquisition of Microdischarge Pulses. IEEE Trans. Diel. El. Insul. Vol. 8, 2001, p. 220-227.

[4] Ficker, T.: Electron Avalanches 1. Statistics of Partial Microdischarges in Their Pre-Streamer Stage. IEEE Trans. Diel. El. Insul. (in press).

[5] Johnson, N. J., Kotz, S.: Continuous Univariate Distributions - I. New York: John Wiley \& Sons, 1970.

Assoc. Prof. RNDr. Tomáš Ficker, DrSc,

phone: + 420541147661

e-mail: fyfic@fce.vutbr.cz

Department of Physics

Assoc. Prof. RNDr. Jiří Macur, CSc.

phone: +420541 147249

e-mail: macur.j@fce.vutbr.cz

Department of Informatics

Faculty of Civil Engineering

University of Technology

Žižkova 17

66237 Brno, Czech Republic

Fig. 2: Probability density of heights of microdischarge pulses [4] 